\title{
Resveratrol decreases high glucose-induced apoptosis in renal tubular cells via suppressing endoplasmic reticulum stress
}

\author{
JING ZHANG ${ }^{1 *}$, XIONG-JUN DONG ${ }^{2 *}$, MENG-RU DING ${ }^{3,4}$, CHUN-YU YOU $^{1}$, XIN LIN $^{1}$, \\ YING WANG $^{5}$, MIAO-JIE-YANG WU ${ }^{5}$, GUO-FEI XU ${ }^{3,5}$ and GUO-DONG WANG ${ }^{3,4}$ \\ ${ }^{1}$ Department of Nephrology, Yijishan Hospital, The First Affiliated Hospital of Wannan Medical College, \\ Wuhu, Anhui 241001; ${ }^{2}$ Department of Nephrology, The Second People's Hospital of Wuhu, Wuhu, Anhui 241000; \\ ${ }^{3}$ Anhui Provincial Engineering Research Center for Polysaccharide Drugs, Anhui Province Key Laboratory of \\ Active Biological Macro-Molecules, Wuhu, Anhui 241002; ${ }^{4}$ School of Pharmacy, Drug Research and \\ Development Center, Wannan Medical College, Wuhu, Anhui 241001; ${ }^{5}$ School of Medical Imaging, \\ Wannan Medical College, Wuhu, Anhui 241002, P.R. China
}

Received April 27, 2020; Accepted August 20, 2020

DOI: $10.3892 / \mathrm{mmr} .2020 .11511$

\begin{abstract}
Diabetic nephropathy (DN) is the second most common complication of diabetes mellitus after cardiovascular complications. Endoplasmic reticulum (ER) stress is known to be associated with DN. Resveratrol (RSV) exhibits anti-oxidative, anti-inflammatory and cytoprotective effects. Therefore, the aims of the present study were to investigate the role of RSV in the inhibition of high concentration glucose (HG)-induced apoptosis in renal tubular cells, as well as to examine the protective effects of RSV against diabetes-mediated renal damage via inhibition of ER stress in DN. RSV was orally administered to diabetic $\mathrm{db} / \mathrm{db}$ mice once a day for 12 consecutive weeks. Compared with untreated $\mathrm{db} / \mathrm{db}$ mice, treating $\mathrm{db} / \mathrm{db}$ mice with RSV significantly decreased urine albumin excretion and the urine albumin to creatinine ratio, and attenuated renal histopathological injury. Furthermore,
\end{abstract}

Correspondence to: Dr Guo-Dong Wang or Dr Guo-Fei Xu, Anhui Provincial Engineering Research Center for Polysaccharide Drugs, Anhui Province Key Laboratory of Active Biological Macro-Molecules, 22 West Wenchang Road, Wuhu, Anhui 241002, P.R. China

E-mail: guodong201@csu.edu.cn

E-mail:20010005@wnmc.edu.cn

${ }^{*}$ Contributed equally

Abbreviations: DN, diabetic nephropathy; RSV, resveratrol; ER, endoplasmic reticulum; DM, diabetes mellitus; CMC, carboxymethyl cellulose sodium salt; BG, blood glucose; BW, body weight; NG, normal glucose; UACR, urine albumin to creatinine ratio; UAE, urine albumin excretion; ROS, reactive oxygen species; GRP78, glucose-regulated protein $78 \mathrm{kD}$; CHOP, C/EBP-homologous protein

Key words: resveratrol, renal tubular cell, apoptosis, diabetic nephropathy, endoplasmic reticulum stress
RSV treatment resulted in decreased expression levels of glucose-regulated protein of $78 \mathrm{kDa}$ and C/EBP-homologous protein (two ER stress markers) and caspase12 in murine kidneys. RSV administration also inhibited the apoptosis of NRK-52E cells and activation of the ER stress signal transduction pathway induced by HG treatment in vitro. Collectively, the present results indicated that RSV protected renal tubular cells against HG-induced apoptosis in DN by suppressing ER stress.

\section{Introduction}

In China, >100 million patients have diabetes mellitus (DM) (1). Diabetic nephropathy (DN), a main complication of DM, is a serious threat to the life and health of Chinese patients and significantly increases medical expenditure (2). Pathological characteristics of DN include glomerular hypertrophy, thickening of the glomerular and tubular basement membrane, deposition of extracellular matrix and finally progression to tubulointerstitial fibrosis and glomerulosclerosis (3). Although it is generally considered that the changes associated with glomeruli serve a key role in the pathogenesis of DN, tubulointerstitial injury may also be an important marker of DN; tubular cells are one of the main targets of high concentration glucose (HG)-induced injury (4). Tubulointerstitial injury is more closely associated with the decline of renal function (5) and can more effectively predict DN progression (6) compared with glomeruli injury. However, the exact role of renal tubular injury in the pathogenesis of DN remains unknown. Increasing evidence has suggested that apoptosis is involved in renal tubular cell damage-related DN (7-9), and endoplasmic reticulum (ER) stress exerts a major role in cell death-related pathways $(10,11)$.

Resveratrol (RSV) is a natural plant polyphenol with anti-oxidative, anti-inflammatory and cytoprotective properties (12). In addition to its antioxidant effect or ability to activate AMP-activated protein kinase or sirtuin 1 (SIRT1) genes (13), RSV has been reported to alleviate the apoptosis 
of hepatocytes (14) and cardiomyocytes (15), as well as neuroinflammation in vasculitic peripheral neuropathy (16) via suppressing ER stress. A previous study also revealed that RSV can attenuate the progression of DN (17). However, to the best of our knowledge, the renoprotective effects of RSV and its association with attenuation of tubular cell injury by inhibiting ER stress-induced apoptosis in DN are yet to be elucidated. The present study performed in vivo and in vitro analyses to determine whether inhibition of ER stress-induced apoptosis by RSV could attenuate tubular cell injury in DN.

\section{Materials and methods}

Animals. Male db/db (C57BLKS/J-LepRdb/LepRdb) mice (average age, 6 weeks; weight 30-33 g; $\mathrm{n}=20$ ) and male $\mathrm{db} / \mathrm{m}$ (C57BLKS/J-LepRdb/+; average age, 6 weeks; weight 20-23 g; $\mathrm{n}=10$ ) were purchased from the National Mode Animal Centre of Nanjing University. All mice were kept at a constant room temperature $\left(20 \pm 1^{\circ} \mathrm{C}\right)$ under a controlled 12-h light/12-h dark cycle in a specific pathogen-free room and allowed to free access to rodent chow and clean water. After adaptive feeding for 2 weeks, all mice were randomly divided into the following groups ( $\mathrm{n}=10 /$ group): $\mathrm{db} / \mathrm{m}$ group, non-diabetic $\mathrm{db} / \mathrm{mmice} ; \mathrm{db} / \mathrm{db}$ group, non-treated db/db mice; and $\mathrm{db} / \mathrm{db}+\mathrm{RSV}$ group, $\mathrm{db} / \mathrm{db}$ mice administered with RSV by gavage. RSV (Sigma-Aldrich; Merck KGaA) was dissolved in carboxymethyl cellulose $(0.5 \%$; CMC; Sigma-Aldrich; Merck KGaA) and orally administered via gavage tube at a dose of $40 \mathrm{mg} / \mathrm{kg}$ once a day for 12 weeks to mice in the $\mathrm{db} / \mathrm{db}+\mathrm{RSV}$ group. The mice in $\mathrm{db} / \mathrm{m}$ and $\mathrm{db} / \mathrm{db}$ groups were administered $100 \mu \mathrm{l} / 10 \mathrm{~g}$ weight of $0.5 \% \mathrm{CMC}$. All animal experimental protocols were ethically approved by the Laboratory Animals Ethical Committee of Wannan Medical College (approval no. LLSC-2020-057). According to the method reported previously (18), mice were anesthetized (fentanyl/medetomidine/midazolam; 0.05/5/0.5 mg/kg body weight; intraperitoneal) at 20 weeks of age, punctured into the abdominal aorta and exsanguinated.

Physical and biochemical analysis. The body weight (BW) of each mouse was measured and blood glucose (BG) from the tail vein blood $(50 \mu \mathrm{l})$ was tested with a glucometer (Accu-Check Active; Roche Diagnostics $\mathrm{GmbH}$ ) at both 8 and 20 weeks of age. The mice were placed in metabolic cages to collect 24-h urine samples at 20 weeks of age. The urinary albumin and urinary creatinine concentrations were determined using a mouse albumin ELISA kit (cat. no. E99-134; Bethyl Laboratories, Inc.) and mouse QuantiChrom ${ }^{\mathrm{TM}}$ Creatinine assay kit (cat. no. DICT-500; BioAssay Systems), respectively, according to the manufacturer's protocol.

Histology evaluation. Renal tissues from the mice were fixed for $24 \mathrm{~h}$ at $20^{\circ} \mathrm{C}$ in $10 \%$ neutral formalin, embedded in paraffin, manually sectioned into $4-\mu \mathrm{m}$ thick tissue sections and stained with hematoxylin and eosin (H\&E), periodic acid-Schiff (PAS) and Masson reagent as previously described $(19,20)$.

The paraffin-embedded renal tissue sections were analyzed using immunohistochemistry as described in our previous study (19). Briefly, after dewaxing and hydration, the slides were heated in sodium citrate buffer $(10 \mathrm{mM}$; pH 6) for $10 \mathrm{~min}$ at $100^{\circ} \mathrm{C}$ and incubated with hydrogen peroxide $(0.3 \%)$ for
10 min at room temperature. After blocking with horse serum (Beyotime Institute of Biotechnology) at $37^{\circ} \mathrm{C}$ for $30 \mathrm{~min}$, the sections were stained with primary monoclonal antibodies against GRP78 (1:100; Cell Signaling Technology, Inc.; cat. no. 3177) and CHOP (1:50; Cell Signaling Technology, Inc.; cat. no. 2895) overnight at $4^{\circ} \mathrm{C}$. After washing with Tris-buffered saline containing $0.1 \%$ Tween-20 three times, anti-rabbit and anti-mouse IgG labeled with horseradish peroxidase (Thermo Fisher Scientific, Inc.; cat. nos. 31460 and 31430) were added and the samples were incubated for $45 \mathrm{~min}$ at $20^{\circ} \mathrm{C}$. Images were captured using a light microscope (Nikon Corporation).

TUNEL staining. TUNEL staining was performed using the DeadEnd ${ }^{\mathrm{TM}}$ Colorimetric TUNEL system (Promega Corporation) as described previously (19). Briefly, renal paraffin sections were dewaxed, incubated with proteinase-K (1:500) at $37^{\circ} \mathrm{C}$ for $10 \mathrm{~min}$ and reacted with the TUNEL reaction mixture (balanced solution $98 \mu \mathrm{l}+$ biotinylated nucleotide $\operatorname{mix} 1 \mu \mathrm{l}+\mathrm{rTdT} 1 \mu \mathrm{l}$ ) for $60 \mathrm{~min}$ at $37^{\circ} \mathrm{C}$. The apoptotic cells were counted by two independent pathologists under a light microscope in a blinded manner, and the rate of apoptosis (\%) was determined.

Cell culture experiments. Normal rat renal proximal tubular epithelial (NRK-52E) cells were obtained from the Cell Resource Center of Shanghai Institutes for Biological Sciences, Chinese Academy of Sciences. NRK-52E cells were maintained in DMEM (Gibco; Thermo Fisher Scientific, Inc.) containing 10\% FBS (Gibco; Thermo Fisher Scientific, Inc.) and $1 \%$ penicillin-streptomycin, and incubated in a $5 \% \mathrm{CO}_{2}$ atmosphere at $37^{\circ} \mathrm{C}$.

To test the effect of RSV on HG-induced ER stress, NRK-52E cells were treated with FBS-free medium for $24 \mathrm{~h}$ until the cells reached $\sim 80 \%$ confluency. The cells were subsequently treated with or without $20 \mu \mathrm{M}$ RSV for $6 \mathrm{~h}$ at $37^{\circ} \mathrm{C}$, before incubation with normal concentration glucose (NG; $5.5 \mathrm{mM}$ D-glucose), HG (30 mM D-glucose) or high concentration mannitol (HM, 5.5 mM D-glucose supplied with 24.5 mM D-mannitol; Sigma-Aldrich; Merck KGaA). All tests were performed in triplicate wells and repeated three times.

RNA extraction and reverse transcription-quantitative $(R T-q) P C R$. Total RNA was extracted from NRK-52E cells using TRizol ${ }^{\circledR}$ (Invitrogen; Thermo Fisher Scientifc, Inc.), and the purity and concentration of the extracted RNA were assessed with a spectrophotometer (NanoDrop 2000; Thermo Fisher Scientific, Inc.). RNA was reverse-transcribed with SuperScript III reverse transcriptase (Invitrogen; Thermo Fisher Scientific, Inc.) using an oligo dT primer according to the manufacturer's protocol. RT-qPCR was performed in a StepOne Plus ${ }^{\mathrm{TM}}$ Real-Time PCR system (Applied Biosystems; Thermo Fisher Scientific, Inc.) using a Quanti Nova ${ }^{\mathrm{TM}} \mathrm{SYBR}^{\circledR}$ Green PCR kit (Qiagen $\mathrm{GmbH}$ ). The thermocycling conditions consisted of an initial denaturation at $95^{\circ} \mathrm{C}$ for $10 \mathrm{~min}$ followed by 40 cycles of $95^{\circ} \mathrm{C}$ for $15 \mathrm{sec}$ and $60^{\circ} \mathrm{C}$ for $60 \mathrm{sec}$. The following primers were used: i) GRP78-forward, 5'-GAC TGGAATCCCTCCTGCTC-3' and reverse, 5'-GGTCAG GCGGTTTTGGTC-3'; ii) CHOP-forward, 5'-CACAAGCAC CTCCCAAAGC-3' and reverse, 5'-CTCTCATTCTCCTGC 
A

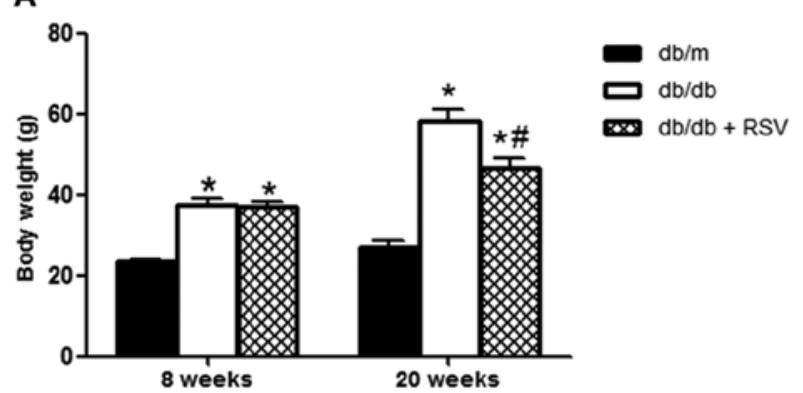

C

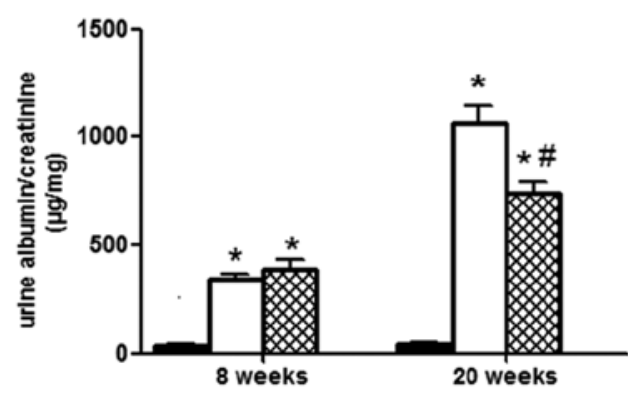

B

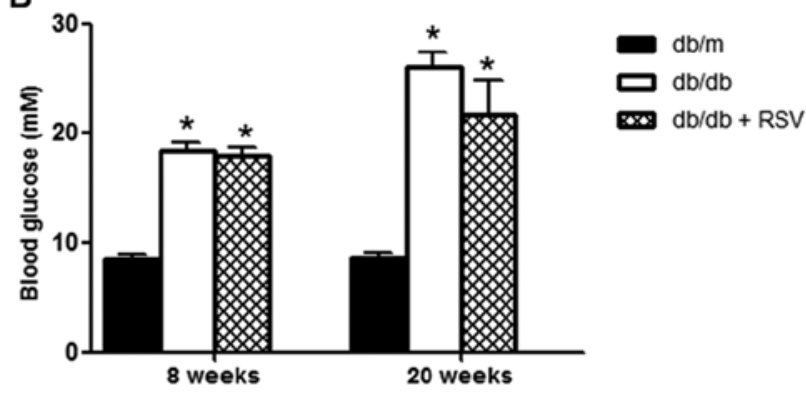

D

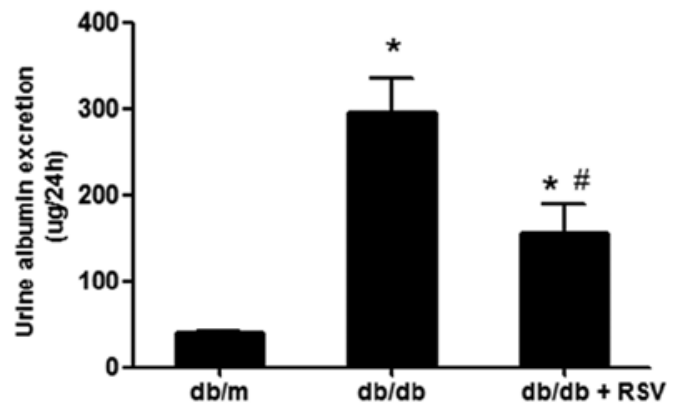

Figure 1. RSV attenuates renal injury in $\mathrm{db} / \mathrm{db}$ mice. (A) Body weight, (B) blood glucose, (C) urine albumin and creatinine ratio and (D) urine albumin excretion levels in $\mathrm{db} / \mathrm{db}$ mice were decreased by RSV treatment. Data are presented as the mean $\pm \mathrm{SEM}$. "P $<0.05 \mathrm{vs}$. db/m group; ${ }^{\#} \mathrm{P}<0.05 \mathrm{vs}$. db/db group. RSV, resveratrol.

TCCTTCTC-3'; and iii) GAPDH-forward, 5'-ACTCCACGA CATACTCAGCA-3' and reverse, 5'-CATCAACGACCCCTC ATT-3'. mRNA expression levels were normalized to those of GAPDH in the same cDNA sample. Relative quantification of gene expression was performed using the $2^{-\Delta \Delta \mathrm{Cq}}$ method (21).

Western blotting. Tissues or cells were lysed with RIPA buffer (Beyotime Institute of Biotechnology) containing protease and phosphatase inhibitor cocktail, and the protein concentration was measured using the bicinchoninic acid protein assay kit (Bio-Rad Laboratories, Inc.). The protein samples (40 $\mu \mathrm{g}$ per lane for tissue; $30 \mu \mathrm{g}$ per lane for cells) were separated via SDS-PAGE on $10 \%$ gels and transferred to PVDF membranes, as described in our previous study (19). After blocking with PBST containing $3 \% \mathrm{BSA}$ for $1 \mathrm{~h}$ at room temperature, the membranes were incubated with the following primary antibodies overnight at $4^{\circ} \mathrm{C}$ : i) GRP78 (1:1,000; Cell Signaling Technology, Inc.; cat. no. 3177), CHOP (1:1,000; Cell Signaling Technology, Inc.; cat. no. 2895); ii) cleaved caspase12 (1:1,000; ABclonal Biotech Co., Ltd.; cat. no. A0217); and iii) $\beta$-actin (1:1,000; Wuhan Boster Biological Technology, Ltd.; cat. no. BA2305). Following incubation, the PVDF membranes were washed with PBS containing $0.1 \%$ Tween-20 and incubated with horseradish peroxidase-conjugated goat anti-rabbit (1:4,000; cat. no. 31460) or goat anti-mouse IgG secondary antibodies (1:4,000; cat. no. 31430) (both Thermo Fisher Scientific, Inc.) at room temperature for $1 \mathrm{~h}$. Protein bands were visualized using electrochemiluminescence western blotting detection reagent (Beyotime Institute of Biotechnology) and scanned using a Bio-Rad Imaging system (version 2.0; Bio-Rad Laboratories, Inc.). ImageJ software (version 1.8.0; National Institutes of Health) was used for analysis.
Cell apoptosis assay. FITC Annexin V Apoptosis Detection Kit I (BD Biosciences; cat. no. 556547) was used to quantify the rate of apoptosis (at early phase) according to the manufacturer's protocols. Briefly, the harvested NRK-52E cells were resuspended in $100 \mu \mathrm{l}$ binding buffer, followed by staining with $5 \mu \mathrm{l}$ annexin V-FITC and $1 \mu \mathrm{g} / \mathrm{ml}$ PI solutions in the dark at room temperature for $15 \mathrm{~min}$. Data were acquired with a Gallios flow cytometer (Beckman Coulter, Inc.) and analyzed using FlowJo software (version 10; FlowJo LLC).

Statistical analysis. Statistical analyses were performed using SPSS 17.0 (SPSS, Inc.). Data are presented as the mean \pm SEM. Multiple groups was compared using one-way ANOVA followed by Tukey's post hoc test. $\mathrm{P}<0.05$ was considered to indicate a statistically significant difference.

\section{Results}

$R S V$ attenuates renal injury and improves renal morphology in diabetic $\mathrm{db} / \mathrm{db}$ mice. The present study determined BG, BW, urine albumin to creatinine ratio (UACR) and urine albumin excretion (UAE) of the mice in each group at 8 and 20 weeks (Fig. 1). Compared with $\mathrm{db} / \mathrm{m}$ mice, $\mathrm{db} / \mathrm{db}$ mice had significant increases in BW, BG, UACR and UAE at 8 and 20 weeks. However, after treatment with RSV, BW, UACR and UAE were significantly decreased at 20 weeks. Although BG improved after RSV treatment, the change was not significantly different.

The characteristic renal histopathological changes were observed via H\&E, PAS and Masson staining in diabetic $\mathrm{db} / \mathrm{db}$ mice at 20 weeks. Compared with the healthy kidney structure in the $\mathrm{db} / \mathrm{m}$ group, mesangial cell proliferation, accumulation and expansion of focal mesangial matrix and tubulointerstitial fibrosis were observed in the $\mathrm{db} / \mathrm{db}$ group. These changes 

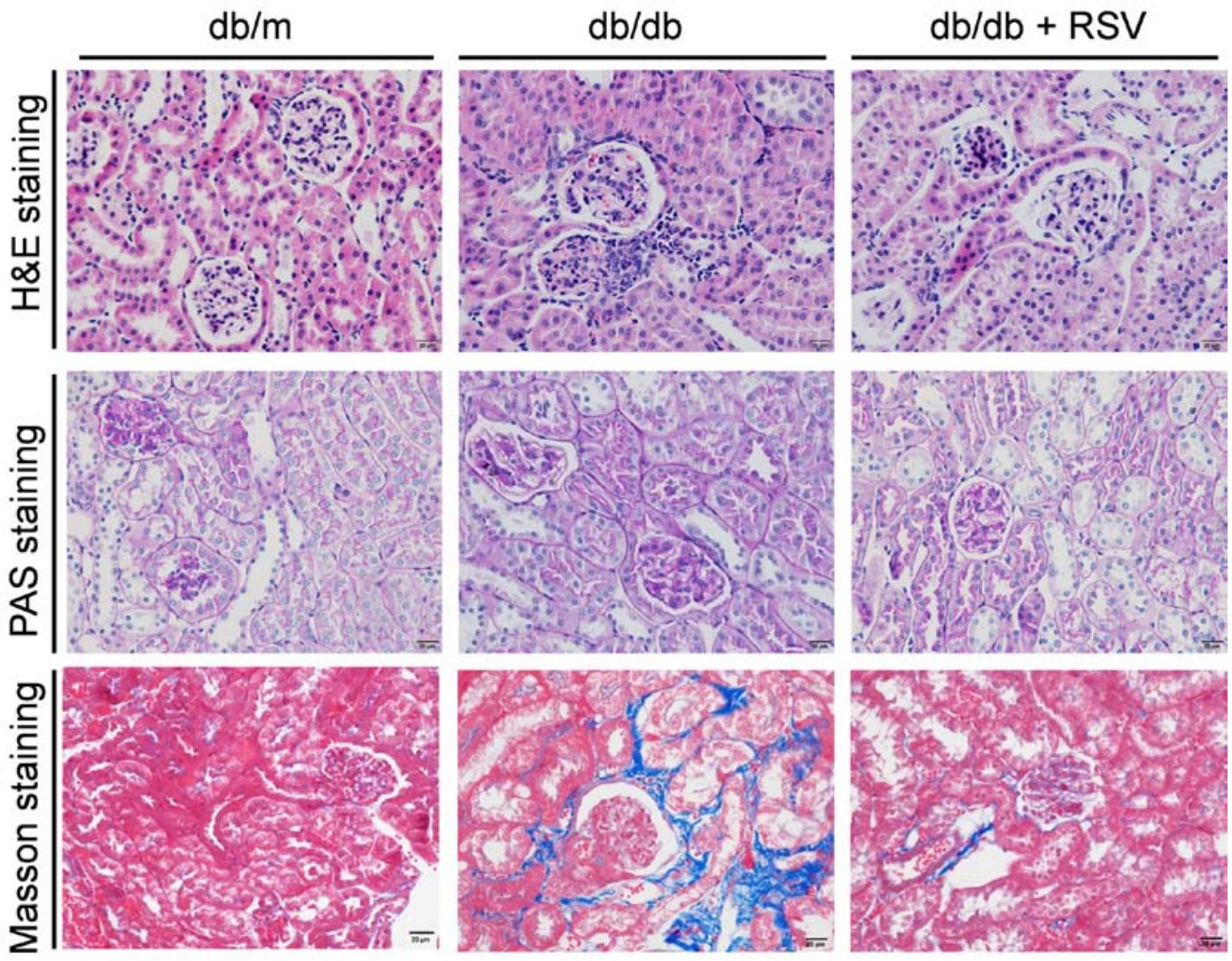

Figure 2. Histological morphology of the kidney. Representative images of H\&E, PAS and Masson-stained renal tissue sections in each group at 20 weeks of age. Scale bar, $20 \mu \mathrm{m}$. H\&E, hematoxylin and eosin; PAS, periodic acid-Schiff; RSV, resveratrol.
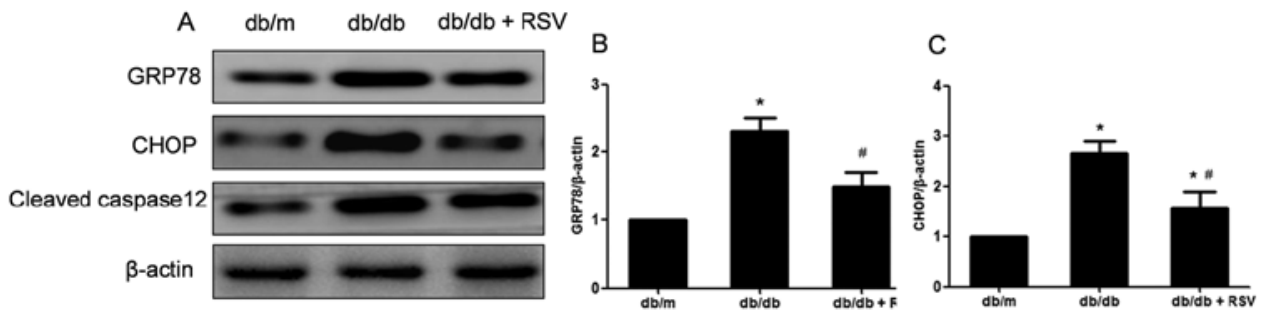

D

Figure 3. RSV suppresses ER stress-induced apoptosis in the kidneys of db/db mice. (A) Protein expression levels of GRP78, CHOP, cleaved caspase12 and $\beta$-actin were measured using western blotting. Semi-quantification of protein expression levels of (B) GRP78, (C) CHOP and (D) cleaved caspase12. Data are presented as the mean \pm SEM. ${ }^{~} \mathrm{P}<0.05 \mathrm{vs}$. db/m group; ${ }^{~} \mathrm{P}<0.05 \mathrm{vs}$. db/db group. RSV, resveratrol; GRP78, glucose-regulated protein of $78 \mathrm{kDa}$; $\mathrm{CHOP}$, C/EBP-homologous protein.

were notably attenuated in the RSV-treated group compared with those in non-treated $\mathrm{db} / \mathrm{db}$ mice (Fig. 2). These results indicated that RSV has beneficial effects in delaying the development of DN.

RSV inhibits ER stress in the kidneys of diabetic db/db mice. ER stress has been reported to be involved in the pathogenesis of DN (22). Therefore, the expression levels of GRP78, CHOP and cleaved caspase 12 were measured in the kidneys of $\mathrm{db} / \mathrm{db}$ mice. The expression levels of GRP78, CHOP and cleaved caspase 12 in the renal cortex of $\mathrm{db} / \mathrm{db}$ mice were significantly higher than those in non-diabetic $\mathrm{db} / \mathrm{m}$ mice. Treatment of $\mathrm{db} / \mathrm{db}$ mice with RSV significantly downregulated the expression levels of GRP78, CHOP and cleaved caspase 12 (Fig. 3). The expression levels of GRP78 and CHOP in the renal tubules of diabetic $\mathrm{db} / \mathrm{db}$ mice were significantly higher, compared with those in non-diabetic $\mathrm{db} / \mathrm{m}$ mice. Downregulation of GRP78 and CHOP in the renal tubule of diabetic $\mathrm{db} / \mathrm{db}$ mice after RSV treatment was also demonstrated via immunohistochemistry (Fig. 4). Thus, the results suggested that ER stress was induced in the kidneys of diabetic $\mathrm{db} / \mathrm{db}$ mice, particularly in the tubules, and was significantly inhibited by RSV treatment.

RSV inhibits ER stress-induced apoptosis in the kidneys of $d b / d b$ mice. ER stress-induced apoptosis serves an important role in cell death (23). The results of TUNEL assay identified that the number of apoptotic renal cells in $\mathrm{db} / \mathrm{db}$ mice was higher compared with those in $\mathrm{db} / \mathrm{m}$ mice, which was significantly decreased by RSV treatment (Fig. 5). Furthermore, western blotting was performed to confirm whether apoptosis 
A

$\mathrm{db} / \mathrm{m}$
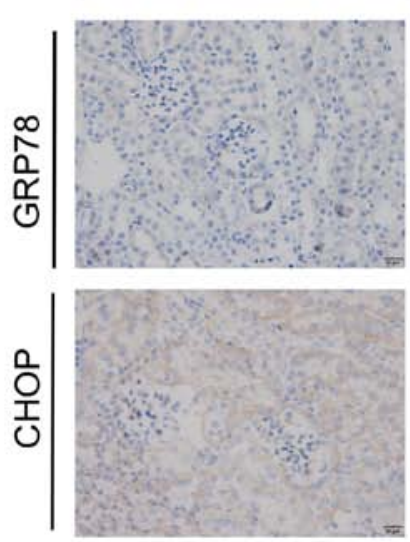

$\mathrm{db} / \mathrm{db}$
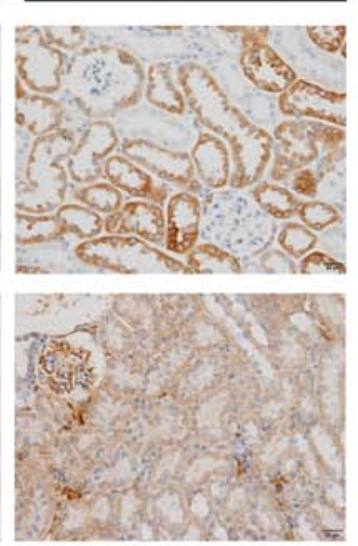

$d b / d b+R S V$
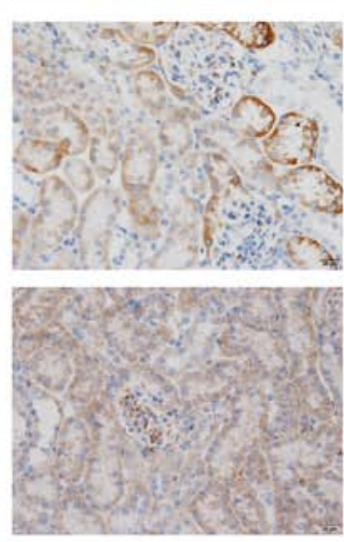

B

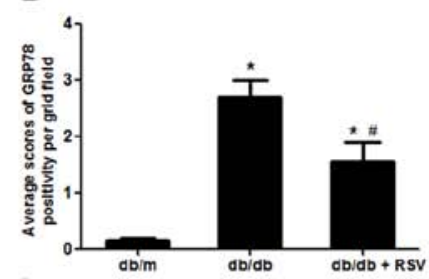

C

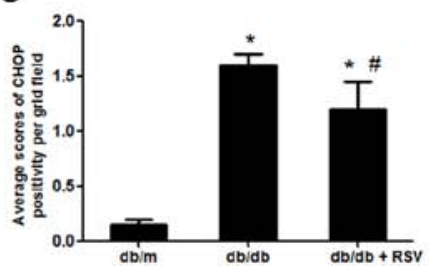

Figure 4. RSV inhibits GRP78 and CHOP expression levels in the kidneys of db/db mice. (A) GRP78 and CHOP expression levels were determined via immunohistochemical staining in the renal tissues of mice in each group. Scale bar, $20 \mu \mathrm{m}$. Semi-quantitative bar graphs of renal (B) GRP78 and (C) CHOP in different groups. Data are presented as the mean \pm SEM. ${ }^{~} \mathrm{P}<0.05 \mathrm{vs}$. db/m group; ${ }^{\prime} \mathrm{P}<0.05 \mathrm{vs}$. db/db group. RSV, resveratrol; GRP78, glucose-regulated protein of $78 \mathrm{kDa}$; CHOP, C/EBP-homologous protein.

A

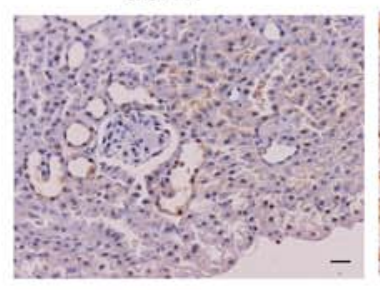

$\mathrm{db} / \mathrm{db}$

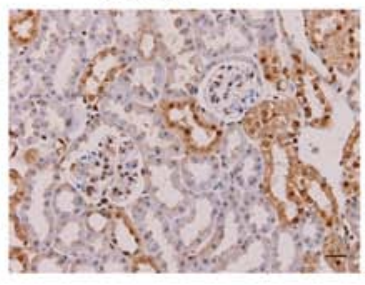

$\mathrm{db} / \mathrm{db}+\mathrm{RSV}$

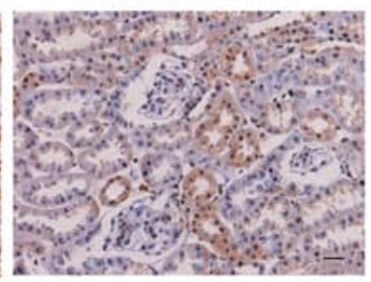

B

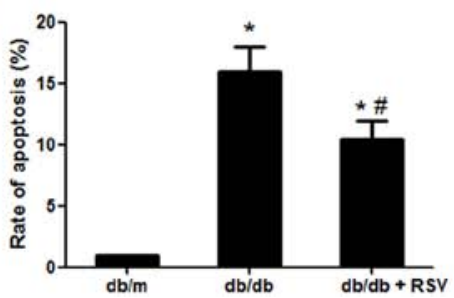

Figure 5. RSV decreases the apoptotic rate in renal cells of db/db mice. (A) Representative images of apoptotic renal cells in mice of each group determined using TUNEL assay. (B) Semi-quantitative bar graphs of TUNEL-positive stained tubular cells in different groups. Scale bar, $20 \mu \mathrm{m}$. Data are presented as the mean $\pm \mathrm{SEM}$. ${ }^{\mathrm{P}}<0.05 \mathrm{vs}$. db/m group; ${ }^{*} \mathrm{P}<0.05$ vs. db/db group. RSV, resveratrol.

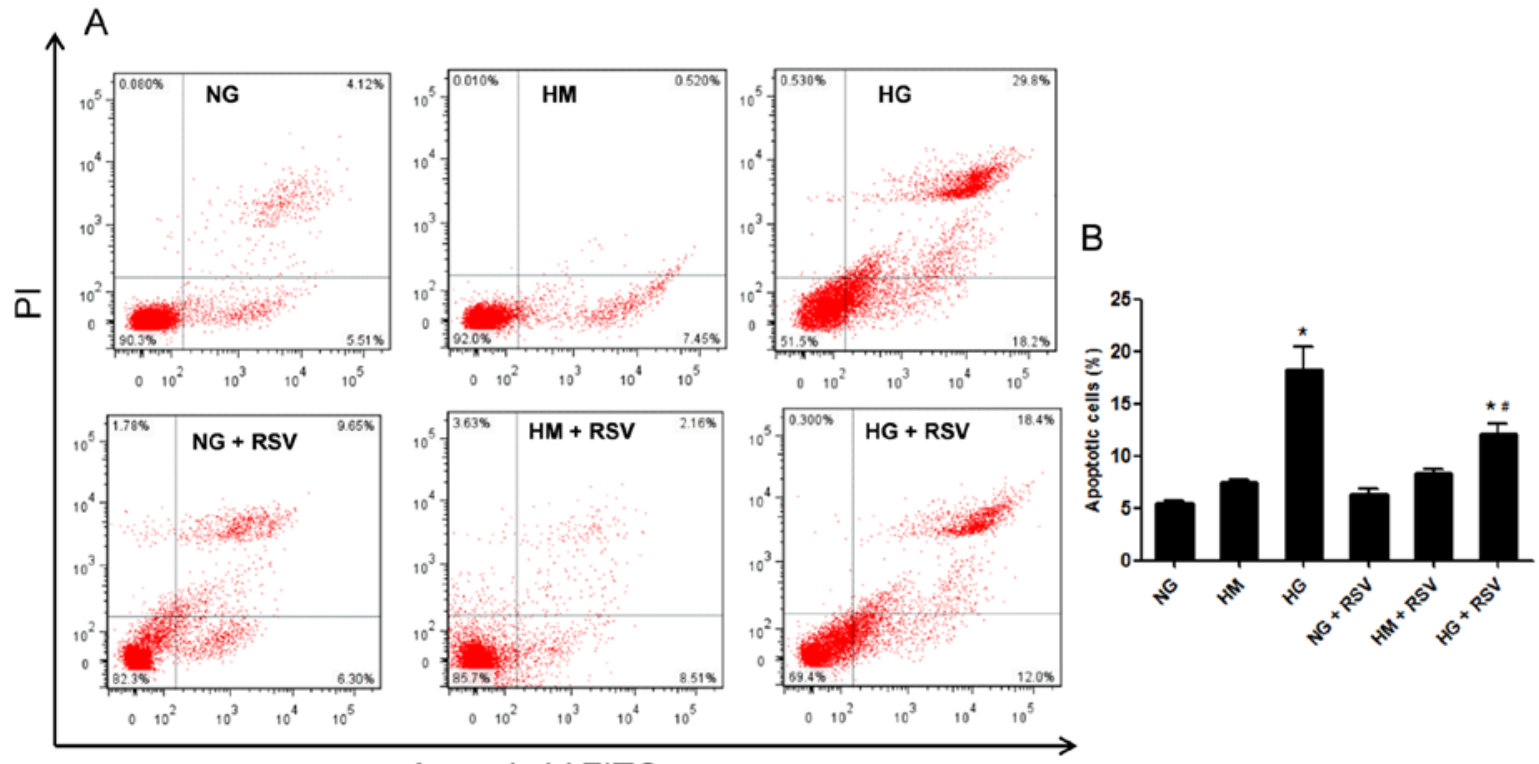

Annexin V-FITC

Figure 6. RSV inhibits high concentration glucose-induced apoptosis in NRK-52E cells. NRK-52E cells were pretreated with $20 \mu \mathrm{M}$ RSV for $6 \mathrm{~h}$, followed by incubation with NG $(5.5 \mathrm{mM})$, HM or HG (30 mM) for another $24 \mathrm{~h}$. (A) Representative images of apoptotic NRK-52E cells determined via flow cytometry. (B) Quantification of apoptotic NRK-52E cells. Data are presented as the mean \pm SEM. ${ }^{*} \mathrm{P}<0.05$ vs. NG group " $\mathrm{P}<0.05$ vs. HG group. RSV, resveratrol; NG, normal glucose; HG, high glucose; HM, high concentration mannitol. 

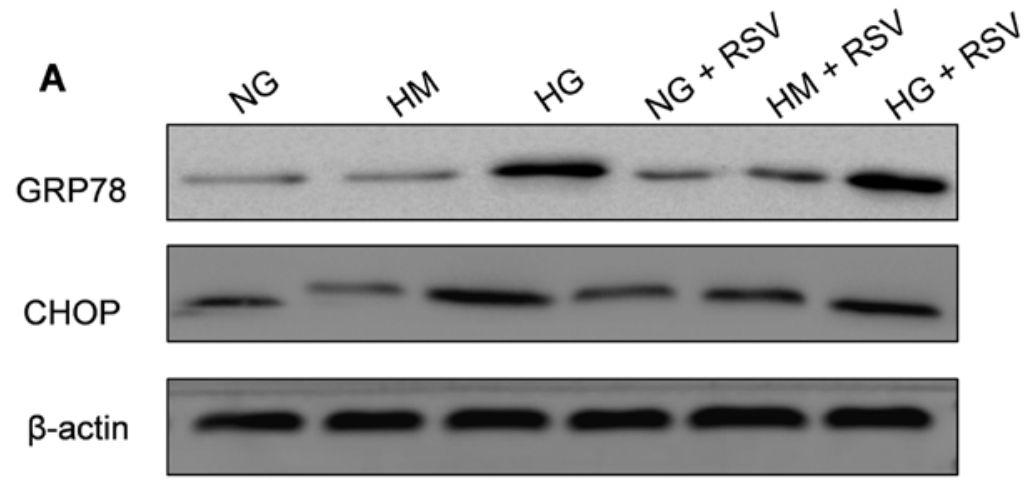

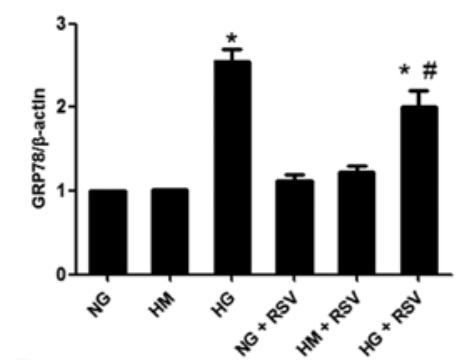

B

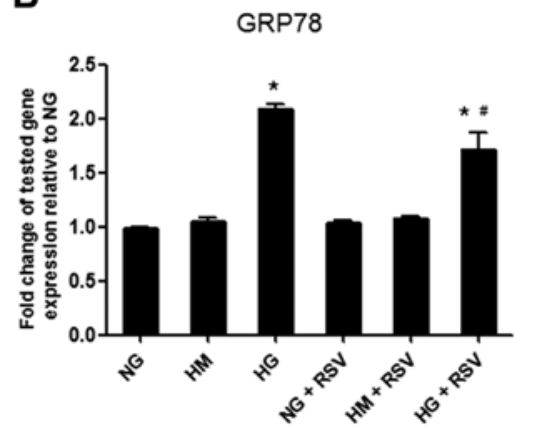

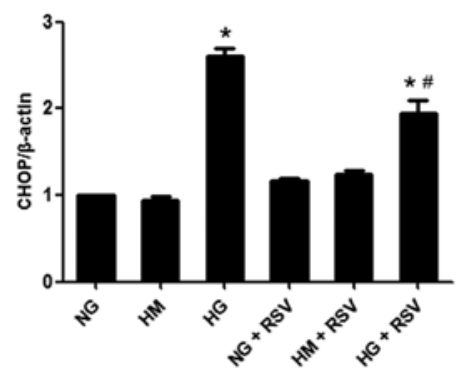

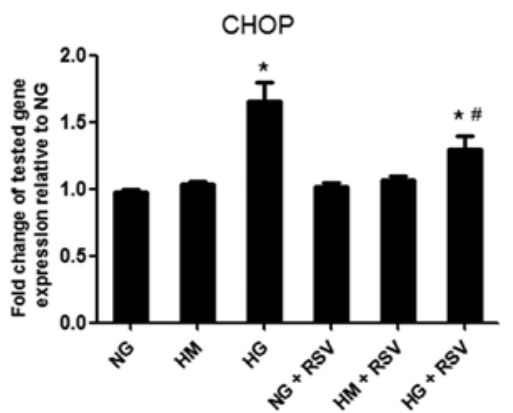

Figure 7. RSV prevents high concentration glucose-induced ER stress in NRK-52E cells. NRK-52E cells were pretreated with $20 \mu \mathrm{M}$ RSV for $6 \mathrm{~h}$, followed by incubation with NG $(5.5 \mathrm{mM})$, HM or HG $(30 \mathrm{mM})$ for another $24 \mathrm{~h}$. The expression levels of GRP78 and CHOP in NRK-52E cells were determined via (A) western blotting and (B) reverse transcription-quantitative PCR. Data are presented as the mean \pm SEM. ${ }^{\text {"P }}<0.05$ vs. NG group ${ }^{\#} \mathrm{P}<0.05$ vs. HG group. RSV, resveratrol; NG, normal glucose; HG, high glucose; HM, high concentration mannitol; GRP78, glucose-regulated protein of $78 \mathrm{kDa}$; CHOP, C/EBP-homologous protein.

was induced by ER stress. The expression of cleaved caspase 12 was significantly increased in the kidney tissues of $\mathrm{db} / \mathrm{db}$ mice compared with $\mathrm{db} / \mathrm{m}$ mice, which was significantly decreased after RSV treatment (Fig. 3D). Therefore, RSV treatment may alleviate ER stress-induced cell apoptosis.

$R S V$ inhibits HG-induced apoptosis in NRK-52E cells. The effects of RSV in vitro were validated using rat renal cells treated with HG. The results demonstrated that HG-induced apoptosis of NRK-52E cells was significantly inhibited by RSV treatment (Fig. 6). These data suggested that HG could induce apoptosis of NRK-52E cells compared with normal concentration glucose, and that RSV could alleviate apoptosis of NRK-52E cells induced by HG.

RSV attenuates HG-induced ER stress in NRK-52E cells. The role of ER stress in inducing apoptosis of NRK-52E cells treated with HG was subsequently investigated. Compared with NG, HG upregulated the expression of ER stress-related proteins GRP78 and CHOP, while HM did not effectively activate ER stress (Fig. 7A). Furthermore, pretreating
NRK-52E cells with RSV at $20 \mu \mathrm{M}$ for $6 \mathrm{~h}$ significantly inhibited the upregulation of GRP78 and CHOP expression levels in NRK-52E cells exposed to HG. Consistent with the western blotting results, the gene expression levels of GRP78 and CHOP in NRK-52E cells cultured with HG were upregulated, which was inhibited by RSV (Fig. 7B). Collectively, it was indicated that ER stress was induced in HG-stimulated NRK-52E cells and RSV effectively suppressed ER stress.

\section{Discussion}

The present results demonstrated that RSV administration can delay the development of DN, as indicated by decreases in UACR and UAE, improvements in the renal histopathology of $\mathrm{db} / \mathrm{db}$ mice and inhibition of ER stress-induced apoptosis. Moreover, RSV inhibited HG-induced ER stress and reduced the rate of apoptosis in tubular cells in vitro. Thus, the present results suggested that RSV reduced HG-induced apoptosis in renal tubular cells by suppressing ER stress.

Renal tubular cells are direct targets of enhanced glucose levels in patients with diabetes, and renal tubular injury can 
precede microalbuminuria (24). HG stimulates renal proximal tubular cells and promotes the production of various cytokines, growth factors, reactive oxygen species (ROS) and matrix proteins (24), which in turn results in tubular hypertrophy and tubular basement membrane thickening (25). Furthermore, HG-induced ROS production results in apoptosis of pancreatic $\beta$-cells by targeting SIRT1 (26), an enzyme that regulates antioxidant-related genes. RSV has been revealed to attenuate several types of renal injury, including DN, drug-induced renal damage, obstructive nephrology, ischemia-reperfusion and sepsis-induced renal damage, in animal models via its antioxidant effect or SIRT1 activation (13). A previous study reported the effect of antioxidants on ER stress (27), which prompted the present study to evaluate the inhibitory role of RSV on ER stress in HG-induced tubular cell injury in DN.

The present findings are in line with those of a recent report by Yuan et al (28), who found that RSV treatment improved diabetes-induced renal damage and decreased ER stress-related markers in streptozotocin-induced diabetic rats. The current study identified that the expression levels of GRP78, CHOP and caspase 12 were downregulated in the kidney cortex of diabetic $\mathrm{db} / \mathrm{db}$ mice administered RSV. Under physiological conditions, GRP78, an important ER chaperone, forms a complex with three transmembrane proteins to maintain the inactive state of ER (29). GRP78 is also a key regulatory factor in unfolded protein response (29). CHOP, a transcription factor, is involved in ER stress-induced cell apoptosis; therefore, it is considered a proapoptotic protein (30). CHOP has been reported to be upregulated during ER stress and is closely associated with the onset of ER stress-induced apoptosis (31). Moreover, CHOP deletion protects cells against ER stress-induced injury (30). The present results demonstrated a downregulation of $\mathrm{CHOP}$ in RSV-treated mice, which was accompanied by decreased apoptosis of renal cells, particularly tubular cells. Caspase12 has been shown to be specifically localized on the ER $(32,33)$ in rodents and is considered a specific sign of ER stress-induced apoptosis $(33,34)$. During the ER stress-induced apoptosis cascade, caspase 12 is cleaved and subsequently activated to induce cell death (35). The present results indicated that RSV treatment can alleviate ER stress-induced renal cell apoptosis by modulating GRP78, CHOP and caspase12 gene expression levels.

The effect of RSV on lowering blood glucose levels in $\mathrm{db} / \mathrm{db}$ mice remains controversial. Previous studies have shown that RSV administration decreases blood glucose levels in $\mathrm{db} / \mathrm{db}$ mice $(36,37)$, whereas recent reports demonstrated no such effect $(38,39)$. The present study found that RSV lowered blood glucose levels in $\mathrm{db} / \mathrm{db}$ mice compared with those in non-treated $\mathrm{db} / \mathrm{db}$ mice; however, the difference was not significant. Therefore, lowering of blood glucose levels may not account for the renoprotective effects of RSV in diabetic mice. Furthermore, in vitro experimental results demonstrated that RSV effectively protected NRK-52E cells against HG-induced apoptosis, suggesting that the renoprotective effects of RSV do not occur via lowering of blood sugar.

Different mechanisms, such as the RAGE/p22phox/ $\mathrm{NF}-\kappa \mathrm{B}$ pathway (40), oxidative stress/TRAF3 interacting protein $2 / \mathrm{NF}-\kappa \mathrm{B}$ pathway (41) and TGF- $\beta 1 / \mathrm{Smad} 2 / 3$ signaling pathway (42), have been reported to be associated with
HG-induced tubular damage. The involvement of ER stress in tubular injury was found to be mainly due to acute kidney injury $(43,44)$. However, in our previous study, it was demonstrated that tauroursodeoxycholic acid, an effective inhibitor of ER stress, attenuated renal tubular injury in a mouse model of type 2 diabetes by suppressing the ER stress signaling pathway (19). In agreement with these previous findings, the present results indicated that tubular cell injury in DN was associated with ER stress. Thus, suppressing HG-induced ER stress and associated apoptosis using RSV treatment may reduce the death of tubular cells.

The present study demonstrated that RSV can inhibit diabetes-induced ER stress in vivo, which was consistent with the finding of a previous study in which RSV treatment inhibited tunicamycin-induced ER stress in vivo (45), indicating a direct inhibitory effect of RSV. HG activates NADPH oxidase, resulting in the production of ROS, which in turn induces ER stress (46). In such cases, suppression of ER stress via RSV may be attributed to its indirect inhibitory effects on oxidative stress. Future studies will further examine the relevant mechanisms in the role of RSV in DN via experiments, such as animal models with gene knockouts, as well as cell models and rescue experiments.

In conclusion, the present study indicated that RSV exerted renoprotective effects in vivo, reduced HG-induced tubular cell apoptosis in vitro and suppressed ER stress. Furthermore, the current study provides evidence for the clinical application of RSV in preventing the development of DN.

\section{Acknowledgements}

Not applicable.

\section{Funding}

This work was supported by the Funding of Yijishan Hospital (grant no. KY24560348), the Scientific Research Project of Wannan Medical College (grant no. WK2019F15). Anhui Provincial Natural Science Foundation (grant no. 1908085MH248), the Overseas Visiting and Training Program for the Outstanding Young Talents in the Colleges of Anhui Province (grant no. gxgwfx2018054) and National Training Programs of Innovation and Entrepreneurship for Undergraduates (grant nos. 201810368004 and 201910368019).

\section{Availability of data and materials}

The datasets used and/or analyzed during the present study are available from the corresponding author upon reasonable request.

\section{Authors' contributions}

JZ, GFX and GDW designed the study. JZ, XJD, MRD and CYY performed the experiments. YW and MJYW helped to feed and prepare animals, and also performed some of the biochemical kit measurements. JZ, XL and GFX analyzed the data. JZ, XJD, GFX and GDW wrote and edited the manuscript. All authors read and approved the manuscript and agree to be accountable for all aspects of the research. 


\section{Ethics approval and consent to participate}

All animal experimental protocols were approved by the Laboratory Animals Ethical Committee of Wannan Medical College (approval no. LLSC-2020-057).

\section{Patient consent for publication}

Not applicable.

\section{Competing interests}

The authors declare that they have no competing interests.

\section{References}

1. Xu Y, Wang L, He J, Bi Y, Li M, Wang T, Wang L, Jiang Y, Dai M, $\mathrm{Lu}$ J, et al: Prevalence and control of diabetes in Chinese adults. JAMA 310: 948-959, 2013.

2. Mao W, Yip CW and Chen W: Complications of diabetes in China: Health system and economic implications. BMC Public Health 19: 269,2019

3. Pourghasem M, Shafi H and Babazadeh Z: Histological changes of kidney in diabetic nephropathy. Caspian J Intern Med 6: 120-127, 2015.

4. Jenkin KA, McAinch AJ, Zhang Y, Kelly DJ and Hryciw DH: Elevated cannabinoid receptor 1 and $\mathrm{G}$ protein-coupled receptor 55 expression in proximal tubule cells and whole kidney exposed to diabetic conditions. Clin Exp Pharmacol Physiol 42: 256-262, 2015.

5. Xiao L, Wang M, Yang S, Liu F and Sun L: A glimpse of the pathogenetic mechanisms of Wnt $/ \beta$-catenin signaling in diabetic nephropathy. Biomed Res Int 2013: 987064, 2013.

6. Gilbert RE and Cooper ME: The tubulointerstitium in progressive diabetic kidney disease: More than an aftermath of glomerular injury? Kidney Int 56: 1627-1637, 1999.

7. Ju Y, Su Y, Chen Q, Ma K, Ji T, Wang Z, Li W and Li W: Protective effects of Astragaloside IV on endoplasmic reticulum stress-induced renal tubular epithelial cells apoptosis in type 2 diabetic nephropathy rats. Biomed Pharmacother 109: 84-92, 2019

8. Shibusawa R, Yamada E, Okada S, Nakajima Y, Bastie CC Maeshima A, Kaira K and Yamada M: Dapagliflozin rescues endoplasmic reticulum stress-mediated cell death. Sci Rep 9: 9887, 2019.

9. Li H, Zhu X, Zhang J and Shi J: MicroRNA-25 inhibits high glucose-induced apoptosis in renal tubular epithelial cells via PTEN/AKT pathway. Biomed Pharmacother 96: 471-479, 2017.

10. Tian J, Mo J, Xu L, Zhang R, Qiao Y, Liu B, Jiang L, Ma S and Shi G: Scoulerine promotes cell viability reduction and apoptosis by activating ROS-dependent endoplasmic reticulum stress in colorectal cancer cells. Chem Biol Interact 327: 109184, 2020.

11. Jiang M, Wang H, Liu Z, Lin L, Wang L, Xie M, Li D, Zhang J and Zhang R: Endoplasmic reticulum stress-dependent activation of iNOS/NO-NF-KB signaling and NLRP3 inflammasome contributes to endothelial inflammation and apoptosis associated with microgravity. FASEB J, 2020 (Epub ahead of print).

12. Malhotra A, Bath S and Elbarbry F: An organ system approach to explore the antioxidative, anti-inflammatory, and cytoprotective actions of resveratrol. Oxid Med Cell Longev 2015: 803971, 2015.

13. Kitada M and Koya D: Renal protective effects of resveratrol. Oxid Med Cell Longev 2013: 568093, 2013.

14. Liu LQ, Fan ZQ, Tang YF and Ke ZJ: The resveratrol attenuates ethanol-induced hepatocyte apoptosis via inhibiting ER-related caspase-12 activation and PDE activity in vitro. Alcohol Clin Exp Res 38: 683-693, 2014.

15. Guo R, Liu W, Liu B, Zhang B, Li W and Xu Y: SIRT1 suppresses cardiomyocyte apoptosis in diabetic cardiomyopathy: An insight into endoplasmic reticulum stress response mechanism. Int J Cardiol 191: 36-45, 2015.

16. Pan PT, Lin HY, Chuang CW, Wang PK, Wan HC, Lee MC and Kao MC: Resveratrol alleviates nuclear factor-KB-mediated neuroinflammation in vasculitic peripheral neuropathy induced by ischaemia-reperfusion via suppressing endoplasmic reticulum stress. Clin Exp Pharmacol Physiol 46: 770-779, 2019.
17. Yan C, Xu W, Huang Y, Li M, Shen Y, You H and Liang X: HRD1-mediated IGF-1R ubiquitination contributes to renal protection of resveratrol in $\mathrm{db} / \mathrm{db}$ mice. Mol Endocrinol 30: 600-613, 2016.

18. Spegel P, Chawade A, Nielsen S, Kjellbom P and Rutzler M: Deletion of glycerol channel aquaporin-9 (Aqp9) impairs long-term blood glucose control in C57BL/6 leptin receptor-deficient (db/db) obese mice. Physiol Rep 3: e12538, 2015.

19. Zhang J, Fan Y, Zeng C, He L and Wang N: Tauroursodeoxycholic acid attenuates renal tubular injury in a mouse model of type 2 diabetes. Nutrients 8: 589, 2016.

20. Zhang J, Cao P, Gui J, Wang X, Han J, Wang Y and Wang G: Arctigenin ameliorates renal impairment and inhibits endoplasmic reticulum stress in diabetic db/db mice. Life Sci 223: 194-201, 2019.

21. Livak KJ and Schmittgen TD: Analysis of relative gene expression data using real-time quantitative PCR and the 2(-Delta Delta C(T)) method. Methods 25: 402-408, 2001.

22. Fan Y, Lee K, Wang N and He JC: The role of endoplasmic reticulum stress in diabetic nephropathy. Curr Diab Rep 17: 17, 2017.

23. Sano R and Reed JC: ER stress-induced cell death mechanisms. Biochim Biophys Acta 1833: 3460-3470, 2013.

24. Morcos M, Sayed AA, Bierhaus A, Yard B, Waldherr R, Merz W, Kloeting I, Schleicher E, Mentz S, Abd el Baki RF, et al: Activation of tubular epithelial cells in diabetic nephropathy. Diabetes 51: 3532-3544, 2002.

25. Szablewski L: Distribution of glucose transporters in renal diseases. J Biomed Sci 24: 64, 2017.

26. Lin N, Li XY, Zhang HM, Yang Z and Su Q: MicroRNA-199a-5p mediates high glucose-induced reactive oxygen species production and apoptosis in INS-1 pancreatic $\beta$-cells by targeting SIRT1. Eur Rev Med Pharmacol Sci 21: 1091-1098, 2017.

27. Malhotra JD, Miao H, Zhang K, Wolfson A, Pennathur S, Pipe SW and Kaufman RJ: Antioxidants reduce endoplasmic reticulum stress and improve protein secretion. Proc Natl Acad Sci USA 105: 18525-18530, 2008.

28. Yuan D, Liu XM, Fang Z, Du LL, Chang J and Lin SH: Protective effect of resveratrol on kidney in rats with diabetic nephropathy and its effect on endoplasmic reticulum stress. Eur Rev Med Pharmacol Sci 22: 1485-1493, 2018.

29. Cunard $R$ and Sharma K: The endoplasmic reticulum stress response and diabetic kidney disease. Am J Physiol Renal Physiol 300: F1054-F1061, 2011.

30. Marciniak SJ, Yun CY, Oyadomari S, Novoa I, Zhang Y, Jungreis R, Nagata K, Harding HP and Ron D: CHOP induces death by promoting protein synthesis and oxidation in the stressed endoplasmic reticulum. Genes Dev 18: 3066-3077, 2004.

31. Jing G, Wang JJ and Zhang SX: ER stress and apoptosis: A new mechanism for retinal cell death. Exp Diabetes Res 2012: $589589,2012$.

32. Nakagawa T and Yuan J: Cross-talk between two cysteine protease families. Activation of caspase-12 by calpain in apoptosis. J Cell Biol 150: 887-894, 2000.

33. Nakagawa T, Zhu H, Morishima N, Li E, Xu J, Yankner BA and Yuan J: Caspase-12 mediates endoplasmic-reticulum-specific apoptosis and cytotoxicity by amyloid-beta. Nature 403: 98-103, 2000.

34. Szegezdi E, Fitzgerald U and Samali A: Caspase-12 and ER-stress-mediated apoptosis: The story so far. Ann N Y Acad Sci 1010: 186-194, 2003.

35. Hitomi J, Katayama T, Taniguchi M, Honda A, Imaizumi K and Tohyama M: Apoptosis induced by endoplasmic reticulum stress depends on activation of caspase-3 via caspase-12. Neurosci Lett 357: 127-130, 2004.

36. Chang CC, Chang CY, Wu YT, Huang JP, Yen TH and Hung LM: Resveratrol retards progression of diabetic nephropathy through modulations of oxidative stress, proinflammatory cytokines, and AMP-activated protein kinase. J Biomed Sci 18: 47, 2011.

37. Kitada M, Kume S, Imaizumi N and Koya D: Resveratrol improves oxidative stress and protects against diabetic nephropathy through normalization of Mn-SOD dysfunction in AMPK/SIRT1-independent pathway. Diabetes 60: 634-643, 2011.

38. Park HS, Lim JH, Kim MY, Kim Y, Hong YA, Choi SR, Chung S, Kim HW, Choi BS, Kim YS, et al: Resveratrol increases AdipoR1 and AdipoR2 expression in type 2 diabetic nephropathy. J Transl Med 14: 176, 2016. 
39. Xu XH, Ding DF, Yong HJ, Dong CL, You N, Ye XL, Pan ML, $\mathrm{Ma} J \mathrm{~J}$, You Q and Lu YB: Resveratrol transcriptionally regulates miRNA-18a-5p expression ameliorating diabetic nephropathy via increasing autophagy. Eur Rev Med Pharmacol Sci 21: 4952-4965, 2017

40. Yang PY, Li PC and Feng B: Protective effects of gliclazide on high glucose and AGEs-induced damage of glomerular mesangial cells and renal tubular epithelial cells via inhibiting RAGE-p22phox-NF-kB pathway. Eur Rev Med Pharmacol Sci 23: 9099-9107, 2019.

41. Das NA, Carpenter AJ, Belenchia A, Aroor AR, Noda M, Siebenlist U, Chandrasekar B and DeMarco VG: Empagliflozin reduces high glucose-induced oxidative stress and miR-21-dependent TRAF3IP2 induction and RECK suppression, and inhibits human renal proximal tubular epithelial cell migration and epithelial-to-mesenchymal transition. Cell Signal 68: 109506, 2020.

42. Wang Y, Zhang X, Mao Y, Liang L, Liu L, Peng W, Liu H, Xiao Y, Zhang Y, Zhang F, et al: Smad2 and Smad3 play antagonistic roles in high glucose-induced renal tubular fibrosis via the regulation of SnoN. Exp Mol Pathol 113: 104375, 2020.
43. Ferre S, Deng Y, Huen SC, Lu CY, Scherer PE, Igarashi P and Moe OW: Renal tubular cell spliced X-box binding protein 1 (Xbpls) has a unique role in sepsis-induced acute kidney injury and inflammation. Kidney Int 96: 1359-1373, 2019.

44. Huang Z, Guo F, Xia Z, Liang Y, Lei S, Tan Z, Ma L and Fu P. Activation of GPR120 by TUG891 ameliorated cisplatin-induced acute kidney injury via repressing ER stress and apoptosis. Biomed Pharmacother 126: 110056, 2020.

45. Li C, Wang L, Huang K and Zheng L: Endoplasmic reticulum stress in retinal vascular degeneration: Protective role of resveratrol. Invest Ophthalmol Vis Sci 53: 3241-3249, 2012.

46. Ozgur R, Uzilday B, Sekmen AH and Turkan I: The effects of induced production of reactive oxygen species in organelles on endoplasmic reticulum stress and on the unfolded protein response in arabidopsis. Ann Bot 116: 541-553, 2015.

(i) (9) This work is licensed under a Creative Commons Attribution-NonCommercial-NoDerivatives 4.0 International (CC BY-NC-ND 4.0) License. 